\title{
Motivos para Emprender un Negocio por Mujeres Rurales en Guanajuato
}

\section{Rea so ns to Sta rt a Business for Rura I Wo men in Gua na jua to}

\section{MAE Juan Antonio Castellanos Cardona Lic. Diana Landin Campos}

Recepción: 23 de Septiembre del 2016

Aceptación: 21 de Octubre del 2016

Publicación: 16 de Dic iembre del 2016
La investigación plantea el problema de ¿Cuáles son los motivos que tienen las mujeres rurales en el estado de Guanajuato para emprender un negocio?

Se diseñó el modelo: Buscar nuevas oportunidades de ingresos por parte de las mujeres rurales en Guanajuato está en función de mejorar su nivel de vida, experiencia y oportunidades latentes - Oplng $=\mathrm{F}(\mathrm{MNV}+$ $E X+O P)$.

Para la validación de la hipótesis se utilizó un enfoque cuantitativo de tipo exploratoria, descriptiva y correlacional; tiene un diseño de corte transversal. El estudio fue aplicado a un grupo de 100 mujeres emprendedoras rurales de diferentes municipios de Guanajuato, que recibirían un apoyo económico para abrir un negocio en su comunidad.

Palabras clave: motivos, mujer rural, emprendedora, proyectos productivos, perspectiva de género.

\section{Abstract}

The research raises the question of what are the reasons that have rural women in the state of Guanajuato to start a business?

The model was designed: Find new revenue opportunities by rural women in Guanajuato is in terms of improving their standard of living, experience and latent opportunities Oplng $=F(M N V+E X+O P)$.

To validate the hypothesis was used an exploratory quantitative approach, descriptive and correlational; It has a crosssectional design. The study was applied to a group of 100 rural women entrepreneurs from different municipalities of Guanajuato, they would receive financial support to start a business in your community.

Keywords: rural woman, reasons, enterprising, production projects, gender perspective. 


\section{Introducción}

Emprender una empresa representa oportunidades de ingresos propios y fuentes de empleo para la familia y la comunidad inmediata. De acuerdo con El Economista (2015) "México, es el segundo país a nivel mundial en desarrollo de emprendedores. De acuerdo con el Reporte Global de Emprendedores Amway (2014), México presenta una actitud positiva para emprender un negocio, ubicándose en el segundo lugar, empatando con Noruega, a nivel mundial, por debajo de Suecia y arriba de Colombia".

El emprendedurismo social es una reacción económica ante la desigualdad basada en el valor social, cuyo objetivo es que los beneficios de la venta o de la prestación de servicios impacten en el desarrollo de las comunidades.

El emprender es una decisión difícil. Son muchos factores que pueden influir para iniciar un negocio. Esto se vuelve más complejo para el medio rural. Esto lo han vivido más de 1,300 mujeres que han recibido un apoyo económico por parte del gobierno para emprender un negocio en su comunidad.

Sobre esta base se identifica la necesidad de conocer los factores que motivan a las mujeres rurales a emprender un negocio en el estado de Guanajuato. Esto sobre la base que el financiamiento no es uno de los factores puesto que la población encuestada tiene un apoyo de gobierno aprobado, sobre el cual aplicarán esa motivación para ser emprendedora.

El estudio fue aplicado a un grupo de 100 mujeres emprendedoras rurales de diferentes municipios de Guanajuato, que recibirían un apoyo económico para abrir un negocio en su comunidad. La herramienta aplicada fue un cuestionario escrito. Las variables latentes determinadas, de acuerdo al marco teórico, son mejorar el nivel de vida, experiencia y oportunidades latentes. Estos fueron analizados estadísticamente para identificar el problema.

La investigación se determina en un alcance de mujeres rurales del estado de Guanajuato, que tienen aprobado un financiamiento para aperturar un negocio, que han recibido una capacitación para esto. Las limitaciones en torno a esto son las reducidas investigaciones relativas a la motivación de la mujer rural en Guanajuato puesto que se puede observar una gran cantidad de solicitudes a programas de gobierno para este fin, pero sin analizar los factores más básicos.

Los resultados se orientarán en diseñar un modelo que contenga las variables que generan motivación para establecer líneas estratégicas que faciliten el emprendedurismo por parte de mujeres en el medio rural. 


\section{Desarrollo}

Emprender una empresa representa oportunidades de ingresos propios y fuentes de empleo para la familia y la comunidad inmediata. De acuerdo con El Economista (2015) "México, es el segundo país a nivel mundial en desarrollo de emprendedores. De acuerdo con el Reporte Global de Emprendedores Amway (2014), México presenta una actitud positiva para emprender un negocio, ubicándose en el segundo lugar, empatando con Noruega, a nivel mundial, por debajo de Suecia y arriba de Colombia". Aunque de 400 nuevas empresas, sobreviven sólo el $10 \%$ de acuerdo a El Financiero (2014), hablar de emprendedores representa personas con valor, decididos a apostar ser sus propios jefes para tener un mejor estilo de vida y a su ritmo. Son factores fundamentales al momento de tomar la decisión de emprender.

El emprendedurismo social es una reacción económica ante la desigualdad basada en el valor social, cuyo objetivo es que los beneficios de la venta o de la prestación de servicios impacten en el desarrollo de las comunidades.

Los emprendimientos sociales representan un vehículo de cohesión e integración social en comunidades apartadas, marginadas y olvidadas por el Estado. Sobre todo en el ámbito rural mexicano que desde la década de los ochenta se ha sometido a diversos ajustes estructurales para atender la política de libre mercado, de acuerdo con Martínez (2013).

De acuerdo con Buendía-Martínez (2013), la actividad emprendedora es un medio para el desarrollo: por su contribución a la actividad económica, y a la capacidad de innovar y evolucionar de los espacios. En el caso de los países en vías de desarrollo, el desarrollo rural tiene una vital importancia no solo por la necesidad de reestructurar el sector agrícola, máximo generador de empleo y fuente de divisas, sino para lograr la erradicación de la pobreza y el desarrollo sostenible.
Particular importancia tiene en este proceso la generación de emprendedores para acelerar el desarrollo económico en las áreas rurales. La contribución de las mujeres tiene que ver con su participación en el ingreso y la riqueza, así como con la diversificación y el enriquecimiento de la actividad emprendedora y el fortalecimiento de los círculos virtuosos del capital humano y el capital social, elementos, todos ellos, positivos para el desarrollo económico.

Por otro lado, las mujeres constituyen las tres quintas partes de los pobres del mundo de acuerdo al ONUDI (2003). Están limitadas a la educación, empleo y toma de decisiones; aunque basados en datos empíricos se considera que la mujer está teniendo una formación, desarrollo de habilidades y elementos más sólidos para toma de decisiones, que el hombre, resultado de su experiencia directa 0 indirecta por los programas sociales a los cuales es "obligada" a participar por medio de reuniones, comités, capacitaciones, eventos, explicaciones, entre otros. Es por el desempleo, migración y falta de oportunidades locales en el medio rural, lo que ha motivado a las mujeres a emprender proyectos productivos para el sostenimiento de la familia, según con Pérez (2008).

De acuerdo con la "Iniciativa Futuros Iguales" impulsada por el Gobierno de los Estados Unidos de América, al cual se unión México en el 2013, cuyo objetivo es promover la igualdad de género mediante la aplicación de oportunidades... y beneficiarse de manera más amplia del crecimiento económico (Inmujeres, 2014), se busca apoyar las redes de empresarias para mejorar el acceso a mercado, fortalecer los instrumentos de financiamiento ye impulsar la creación de pequeñas y medianas empresas lideradas por mujeres. 
La conformación de grupos de mujeres y el acceso a recursos, así como la participación en proyectos productivos (Arellano, 2009), no son factores determinantes para generar cambios en las relaciones de género sino pueden ser dificultades culturales y sociales para el emprendedurismo rural por parte de las mujeres.

De acuerdo con un estudio de Armenteros, López, Gómez, Guerrero (2015), entre los factores de éxito empresarial de las mujeres la obtención de recursos financieros es la parte más difícil del lanzamiento y crecimiento de una micro, pequeña o mediana empresa.

Los informes de las mujeres emprendedoras en la región latinoamericana, desarrollados bajo la coordinación del BID, refieren que las mujeres tienen su mayor acceso a financiamiento a través de la micro finanzas y en particular mediante el microcrédito, y al mismo tiempo muestran que el acceso al finamiento ocupa la categoría más baja dentro del Índice del Entorno Empresarial para emprendedoras.

En el caso de México con la mayor puntuación del índice, evidencia que el microcrédito tiene mejor desempeño en el apoyo para iniciar negocio por encima de los servicios bancarios e inversión de capital trabajo. Ello se sustenta en la política de la Secretaria de Economía del Gobierno Federal, al reconocer que los micros finanzas constituyen una de las estrategias que permitirá sin importar su lugar de origen, su género o su nivel de ingreso el acceso a oportunidades de desarrollo y al instrumentar diversos programas de apoyo a las iniciativas productivas, donde las mujeres ocuparon el $80,5 \%$ de los beneficiados.

Como el objetivo del trabajo es establecer una relación entre el perfil de las mujeres y el acceso al microcrédito, las mujeres encuestadas en este indagación del sector de comercio e industria, arrojando que el $67 \%$ son propietarias de su empresa, $77 \%$ casadas , con estudios universitarios el $58 \%$ de ellas y con una edad promedio de 47 años, el $67 \%$ pertenecen a una asociación profesional y como dato relevante el $79 \%$ hablan de una mejor calidad de vida y el $84 \%$ de una mejor relación familiar, esto de acuerdo con Martínez (2013).

De acuerdo a lo anterior, se identifica la necesidad de conocer los factores que motivan a las mujeres rurales a emprender un negocio en el estado de Guanajuato. Esto sobre la base que el financiamiento no es uno de los factores puesto que la población encuestada tiene un apoyo de gobierno aprobado, sobre el cual aplicarán esa motivación para ser emprendedora. 
Entre los elementos conceptuales a considerar en la presente investigación son:

\section{Mejorar el nivel de vida.}

En la investigación de Arellano (2009), en la segunda hipótesis se planteó: "La participación en proyectos productivos de las mujeres ha favorecido cambios en el acceso a la educación, salud y la distribución y valoración del trabajo, en cuanto a la equidad en la toma de decisiones, el acceso y control de recursos de los y las integrantes de sus grupos domésticos". Se observaron cambios y avances en cuanto al acceso de las mujeres jóvenes a la educación, puesto que es mayor la cantidad de mujeres que tienen acceso a ella, sin embargo no es atribuible a la participación de las mujeres (madres) en los proyectos, sino a los efectos de la modernidad. En cuanto al acceso a educación, no formal o capacitación de las participantes en los proyectos éste fue limitado.

En términos teóricos con respecto al aprendizaje organizacional de "Mujeres emprendedoras de Benito Juárez" permite observar como una organización alcanza un mayor nivel de desarrollo en términos de convertirse en una organización que ha aprendido a aprender, de acuerdo con Borjas (2014).

Tomando como referencia a May (2014), las tasas de deserción observadas en los grupos de trabajo estudiados, así como el abandono de las actividades productivas por parte de las ex - socias de la empresa "Gustillos" hacen considerar que la relación de género dentro de sus respectivos hogares debe ser monitoreada y manejada de forma que ésta no incida negativamente en el desarrollo humano, social y económico propio de la participación de mujeres rurales en empresas sociales.

Los factores que favorecen la participación de las mujeres en las fases del proceso de elaboración, ejecución y puesta en marcha del proyecto son: su interés y necesidad de generación de ingresos; los apoyos de la familia, de la comunidad, del núcleo agrario y del PROMUSAG, así como la creación de redes de apoyo, de acuerdo a Martínez (2009)

En base a lo expresado por las entrevistadas, uno de los beneficios de participar en el PROMUSAG, es el poder contar con un trabajo que genere ingresos económicos, complementando de esta manera el gasto familiar, lo cual las motivó para involucrarse en este tipo de proyectos.

El emprender un negocio ofrece la posibilidad de generar empleos dignos para la familia lo que permite disponer del tiempo para las actividades domésticas así como tener seguridad en su fuente de empleo, lo cual no está supeditado a otras empresas de la región y sus factores de rotación.

Además el emprender permite mejorar las condiciones de vida, como salud y educación, al tener un ingreso superior y esto a su vez permite asignar mayor presupuesto familiar a conceptos de gastos como es la prevención de la salud o simplemente la atención médica en particulares (esto por los deficientes servicios de salud pública); así como también asignar gastos adicionales para desplazarse a escuelas para continuar los estudios de los hijos principalmente.

En este sentido también es importante la necesidad de las madres de familia de buscar ingresos adicionales (o sustitutos del marido) para incrementar las posibilidades económicas en torno a un ambiente económico en el cual no es suficiente los programas de gobierno que se otorgan a la gente en el medio rural o por los ingresos del campo.

Estos dos factores representan motivaciones importantes para generar el interés de las mujeres rurales en emprender un negocio y mejorar su nivel de vida como se ha planteado en este punto. 


\section{Experiencia.}

La exclusión a la tierra es un factor que ha orillado a la mujer rural a emprender empresas que no requieren la propiedad para la producción primaria, pues las mujeres sólo tienen el $19.8 \%$ de los ejidos en México (Inmujeres, 2014). La experiencia en otros grupos sociales ofrece la confianza y habilidades para emprender un nuevo negocio propio. Las actividades productivas están relacionadas con las domésticas (textil, alimentos, artesanal).

Las mujeres en el medio rural tienen una escolaridad promedio de primaria, lo que representa una limitación al tener que comprender acuerdos y realizar cálculos en la empresa. La capacitación por otra parte, está siendo aplicada a las mujeres por medio de los programas de gobierno, así como las decisiones para inversiones y aplicaciones de apoyos; esto genera un empoderamiento mayor que en el hombre, por su falta de participación social.

En el estudio de Bonomo (2013), menciona que la mayoría las mujeres rurales son casadas, emprenden por necesidad, necesitan un capital mayor; mientras que las mujeres emprendedoras urbanas, en su mayoría lo obtienen de su familia. En cuanto a pertenecer a una familia de emprendedores se da que las mujeres rurales tienen más familia emprendedora y lo hacen habiendo realizado una experiencia anterior, en cambio las urbanas no necesariamente.

Cada vez es mayor la participación de la mujer en proyectos productivos, reuniones de comités, acciones de mejoramiento comunitario, entre otras tareas en las cuales se involucran y por medio de las cuales obtienen una experiencia que el mismo hombre está dejando de lado.

Las capacidades desarrolladas en la gestión de comités sociales como el comedor comunitario, ofrece la experiencia para empoderar a la mujer rural y aplicarlo en la dirección de una empresa.
Martínez (2009), nos menciona que la participación en las reuniones para tomar acuerdos o para llevar a cabo las capacitaciones, les permitía reunirse con otras mujeres de la comunidad fuera de su hogar, y poder intercambiar experiencias entre ellas, y con ello reflexionar sobre sus aportes y tener el reconocimiento las hacen al sentirse "útiles" por desarrollar actividades diferentes a las domésticas, como el gestionar los recursos, los insumos para el proyecto. Esto les genera mayor seguridad en las participantes tanto en el ámbito doméstico como en la comunidad.

El emprender un negocio basado en su experiencia, aunque no tengan estudios formales, está permitiendo a las mujeres rurales sentirse satisfechas por sus logros al alcanzar metas personales como empresariales.

Las mujeres están solicitando apoyos para proyectos productivos, al gobierno principalmente pero también a microfinancieras, en actividades en las cuales tienen el gusto o experiencia en la que se sienten con mayor desenvolvimiento. Actividades domésticas 0 tradiciones familiares la están convirtiendo en una oportunidad de negocio.

Además las múltiples capacitaciones que reciben del gobierno así como de organizaciones de la sociedad civil para el desarrollo rural o local, están permitiendo generar interés en la mujer rural para ser productivas y no solo objetos pasivos en la familia.

Así podemos identificar la necesidad de logro y satisfacción como otros de los factores importantes para realizar acciones de emprendedurismo. 


\section{Oportunidades latentes.}

Las oportunidades comerciales que se presentan en su localidad y vecinas, son percibidas con mayor facilidad por las mujeres en el intercambio de experiencias con otras mujeres. El entorno local es parte del potencial de crecimiento de las empresas rurales.

Yazmin (2010), menciona que en las mujeres jefas de familia prevalece ante todo, el sentido de responsabilidad que tienen para con sus hijos e hijas, su trabajo productivo para satisfacer las necesidades de subsistencia y protección, buscan principalmente "el bienestar de sus hijos e hijas".

Los resultados advierten que están siendo satisfechas las necesidades de subsistencia y protección como resultado de la satisfacción de las mismas necesidades de sus hijos e hijas. Las mujeres no buscan la satisfacción de sus propias necesidades, consiguen satisfacer sus necesidades como beneficio colateral cuando se encargan de satisfacer las necesidades de sus hijos e hijas.

Las necesidades de afecto, participación, libertad, identidad, entendimiento, ocio y creación están dirigidas al beneficio de hijos e hijas, no se satisfacen para sí mismas, no se buscan beneficios para las mujeres jefas de familia, se ven beneficiadas de forma colateral cuando cumplen con su trabajo productivo y reproductivo, sin embargo, su vida, sus esfuerzos e ingresos giran en torno a la maternidad, en torno a su jefatura de familia, son madres, son para los otros y las otras, son de sus hijos e hijas.

De acuerdo con Solana-Villanueva (2014), el programa ha beneficiado a las mujeres en sus necesidades inmediatas, pero ha significado un aumento en sus cargas de trabajo y responsabilidades de organización que muchas veces no pueden sostenerse en el hogar. Su oferta se dirige a las actividades económicas tradicionalmente asignadas a las mujeres, particularmente las del medio rural -como economía de traspatio o artesanías-, sin abordar seriamente la problemática desde una perspectiva teórica consistente y actualizada, según.

Las implicaciones en la "nueva ruralidad" motivan a las mujeres a salir del hogar en busca de ingresos monetarios.

En los programas de gobierno federal - estatal se puede identificar una gran cantidad de apoyos solicitados en torno a abarrotes, estéticas o cibercafés. Esto muestra la necesidad de emprender sobre necesidades locales para cubrir necesidades de consumo o de servicios regulares, los cuales se encarecen al no disponer de ellos sino a distancias alejadas de la comunidad. Esto genera la visión de emprender sobre producto o servicio que no se ofrecen o están a precios muy altos por la falta de competencia.

Con base a capacitaciones o experiencias previas en oficios familiares o talleres, la mujer rural se enfoca a emprender negocios los cuales sean fácil de trabajar o los tiempos de los mismos sean manejables; esto para atender sus actividades de madre o mujer.

Otro bloque de proyectos identificados se puede observar que son para proyectos de pocos recursos o poco capital de trabajo, puesto que los apoyos de gobierno son el concepto que menos apoyan. Por lo que para abrir un negocio analizan este factor.

En un pequeño porcentaje, pero va siendo mayor por la tendencia de la sustentabilidad, los proyectos se están enfocando en aprovechar los recursos locales, principalmente naturales, como son nopal, aves, especies nativos, entre otros.

Por lo anterior se puede identificar qué factores importantes como las oportunidades comerciales o de recursos son motivos para emprender por la mujer rural. 


\section{Definición del problema}

Es por lo anterior que resulta importante identificar los motivos que generan el emprendedurismo en las mujeres rurales en Guanajuato, para definir estrategias que permitan el desarrollo de las empresas rurales con el mejor aprovechamiento de los recursos asignados a los proyectos productivos aprobados, así como también se enfoquen estrategias para dirigir los programas de gobierno para despertar esos intereses que tienen las mujeres emprendedoras.

En este problema hemos eliminado el factor de financiamiento que en otros estudios mencionan que es el primer indicador que motiva el emprender empresas en el medio rural, debido a que al grupo de estudio ya lo han recibido por parte de gobierno lo que permite analizar las condiciones para un modelo en el cual esto no sea lo que motive a la mujer rural.

Esto nos permite tener un inicio conceptual diferente sobre variables relativos a la persona, el interés de mejorar su nivel de vida, y las oportunidades latentes de su entorno local.

Por lo cual se establece como problema de investigación: ¿̇Cuáles son los motivos que tienen las mujeres rurales en el estado de Guanajuato para emprender un negocio? Hipótesis

Buscar nuevas oportunidades de ingresos por parte de las mujeres rurales en Guanajuato, está en función de mejorar su nivel de vida, experiencia y oportunidades latentes.

\section{“Oplng = F (MNV + EX + OP)"}

\section{Variables}

Las variables latentes (o constructos) de estudio son:

- Mejorar el nivel de vida (MNV), es emprender para generar empleos dignos para la familia, mejorar las condiciones de vida como salud y educación.
- $\quad$ Experiencia (EX), es el gusto por una actividad de negocio y la capacitación previa en un oficio.

- Oportunidades latentes (OP), es que no se ofrece un producto o servicio en la comunidad, el negocio es fácil trabajar o de pocos recursos, y aprovechar los recursos locales.

Las variables latentes y manifiestas fueron identificadas de los elementos conceptuales del estado del arte presentado anteriormente. Sobre estos se diseñó la hipótesis a comprobar y presentar en el modelo.

\section{Descripción del Método Muestra e instrumento}

Para la validación de la hipótesis se utilizó un enfoque cuantitativo de tipo exploratoria, descriptiva y correlacional; tiene un diseño de corte transversal, porque las variables fueron evaluadas en un único momento con el fin de descubrir su comportamiento.

La muestra fue seleccionada sobre las beneficiarias del programa mencionado, por conveniencia de forma estratificado por sedes, siendo el tipo de muestreo no probabilístico.

El estudio fue aplicado a un grupo de 100 mujeres emprendedoras rurales de 35 diferentes municipios de Guanajuato, que recibieron un apoyo económico para abrir un negocio en su comunidad en el 2014 y 2015. El instrumento aplicado fue un cuestionario escrito, el cual se aplicó directamente a las mujeres participantes en la capacitación presencial a la que participaron como parte del programa de apoyo.

El instrumento fue validado por medio de la técnica de una sesión científica de consulta de expertos utilizando el método Lawshe (1975), para determinar la validez del instrumento propuesto. Además de considerar la validez de constructo (con la bibliografía consultada), validez de criterio (comparado con el diagnóstico de Inmujeres, 2014), validez de contenido (con la clasificación de las variables de estudio). 
El instrumento utilizado fue clasificado en datos demográficos, tipo de proyecto, nivel de vida, experiencia previa y oportunidades. A partir del punto tres se utilizó una escala de Likert de 5 puntos donde: 1 es totalmente desacuerdo y 5 es totalmente de acuerdo. Los estadísticos descriptivos se presentan en la tabla 1.

Datos importantes sobre las mujeres que respondieron el cuestionario, podemos mencionar:

1. El $75 \%$ son casadas, $13 \%$ solteras. El resto son viudas, divorciadas, madre soltera y otros.

2. El $40 \%$ tienen secundaria, el $26 \%$ primaria, el $13 \%$ preparatoria, el $9 \%$ sin estudios, $8 \%$ con licenciatura.

3. El $54 \%$ son amas de casa en ese momento. El $35 \%$ trabajan por su cuenta. El $9 \%$ son empleadas.

4. El $45 \%$ de proyectos solicitados son para servicios, el $25 \%$ comercio, el $26 \%$ de transformación con valor agregado y $3 \%$ agrícola.

5. El $29 \%$ no tiene experiencia en el proyecto, el $27 \%$ tiene entre 1 a 3 años, el $24 \%$ menos de 1 año, 14\% más de 3 años.

6. El $69 \%$ a la primera vez que solicito fue aprobado su proyecto, el $24 \%$ lo había solicitado una vez, el 3\% lo había solicitado dos veces y otro $3 \%$ hasta tres veces.

\section{Metodología de análisis}

El análisis estadístico utilizado fue el Modelo de Ecuaciones Estructurales, específicamente el Mínimos Cuadrados Parciales (PLS por sus siglas en inglés), el cual como primer paso requiere un Análisis de Componentes Principales y posteriormente la comprobación del modelo con una serie de indicadores estadísticos complejos.

El primer análisis realizado fue por medio del software estadístico SPSS, con la herramienta de Análisis Funcional, con Método de extracción:
Análisis de Componentes principales. En este se presentaron resultados no aceptables, que requerían eliminar algunas variables consideradas en el análisis (var. 6. Tener en la familia un negocio, tipo de proyecto solicitado, veces que se ha solicitado, experiencia y familiar que participan en el proyecto).

A la par se hizo otro análisis con software estadístico SmartPLS, sobre el cual se reflejó el mismo resultado obtenido con el SPSS: eliminar las variables que no aportaban al modelo (ya mencionadas). Por lo cual se decidió eliminarlas del análisis.

Con el ajuste anterior se obtuvo una confiabilidad del instrumento determinado con el coeficiente de Alfa de CronBach, siendo de 0.713 mediante el software estadístico SPSS, superior a un mínimo de 0.70 .

Se hizo la prueba de validez con el análisis funcional, con el mismo software, obteniendo en la prueba de Kaiser-Meyer-Olkin un valor de 0.842 (superior a 0.5 como valor de referencia), en la prueba de Esferidad Barltlett un valor de 0.000 (inferior a 0.05 como referencia) y en todas las comunalidades presentan valores de 0.4 (valor de referencia). La muestra es adecuada al instrumento y existe asociación de ítem con ítem.

Además la carga factorial fue en cuatro componentes de acuerdo a la matriz rotada. Con esta información se procedió a continvar el análisis con el software SmartPLS, más útil para modelado sin supuestos para investigaciones sociales y que permite una muestra pequeña de datos, basado en Modelo Ecvaciones Estructurales (Chin, 1999).

Con SmartPLS se modelo la hipótesis para su comprobación. Los resultados muestran ser aceptables:

1. Confiabilidad de Alfa de Cronbach (presupone a priori que cada indicador de un constructo o variable latente contribuye de la misma forma) superior a 0.50 de las variables latentes, de acuerdo con Jöreskog y Sörbom (1993). Hay consistencia interna de las variables. Ver tabla 2. 
2. La validez convergente lo muestra el AVE (Average Variance Extracted, grado en que los indicadores reflejan la construcción, es decir, si mide lo que pretende medir) superior a 50\%, según Baggozi y Yi (1998). El constructo explica más de la mitad de las varianzas. Ver tabla 2.

3. Existe fiabilidad (consistencia de los indicadores que forman el constructo) con valores mínimos de 0.70. Existe consistencia de los ítems de las variables latentes.
4. Las $R$ cuadrados (miden la cantidad de varianza del constructo que es explicada por el modelo) son superiores a 0.30 y se analiza a la vez los coeficientes de ruta (Beta) para analizar el cumplimiento de la hipótesis (Chin, 1998). Ver tabla 2.

\begin{tabular}{|lrrrrr|}
\hline & Mínimo & Máximo & Media & Desv. Típ. & Varianza \\
Municipio & 1 & 35 & 16.56 & 10.599 & 112.333 \\
Edad & 19 & 74 & 41.43 & 12.099 & 146.393 \\
educativo & 1 & 10 & 8.37 & 2.192 & 4.804 \\
Ocupación & 1 & 7 & 2.98 & 2.329 & 5.424 \\
Estado Civil & 1 & 7 & 2.00 & 1.885 & 3.552 \\
Personas que dependen & 0 & 10 & 3.48 & 1.887 & 3.560 \\
Proyecto & 1 & 5 & 3.70 & 1.188 & 1.412 \\
Experiencia & 1 & 9 & 7.37 & 1.486 & 2.210 \\
Familiares en proyecto & 0 & 10 & 1.98 & 1.517 & 2.302 \\
Veces que solicitan el proyecto & 0 & 4 & 0.37 & .750 & .563 \\
Empleos para la familia 1 & 1 & 5 & 4.64 & .847 & .717 \\
Oportunidad de ingresos 2 & 1 & 5 & 4.22 & .935 & .874 \\
Mejorar la vida 3 & 1 & 5 & 4.41 & .874 & .765 \\
Gusto genera interés 4 & 1 & 5 & 4.64 & .847 & .717 \\
Capacitación da oportunidad 5 & 1 & 5 & 4.61 & .776 & .602 \\
En familia tienen negocio & 1 & 5 & 4.48 & .893 & .797 \\
Producto no hay en comunidad 7 & 1 & 5 & 3.94 & 1.360 & 1.851 \\
Fácil trabajo o bajos rec 8 & 5 & 3.45 & 1.314 & 1.727 \\
Recursos locales 9 & 1 & 4.43 & .856 & .732 \\
\hline
\end{tabular}

Tabla 1. Estadísticos descriptivos de los datos obtenidos con el cuestionario. Elaboración propia.

\begin{tabular}{|l|c|c|c|c|c|}
\hline \multicolumn{1}{|c|}{ Variables latentes } & $\alpha$ Cronbach & AVE & Fiabilidad & $\begin{array}{c}\text { Coeficiente de } \\
\text { ruta }\end{array}$ & $\begin{array}{c}\text { R Cuadra- } \\
\text { da }\end{array}$ \\
\hline Nivel de vida & 0.524 & 0.677 & 0.807 & 0.346 & \\
\hline Experiencia & 0.724 & 0.783 & 0.878 & 0.127 & \\
\hline Oportunidades & 0.596 & 0.541 & 0.777 & 0.350 & \\
\hline Oportunidades de ingreso & 1.000 & 1.000 & 1.000 & & 0.402 \\
\hline
\end{tabular}

Tabla 2. Resumen de resultados con el SmartPLS. Elaboración propia. 
Results

- Cronbach's Alpha = 0.724

- Composite Reliability $=0.878$

- Average Variance Extracted (AVE) $=0.783$

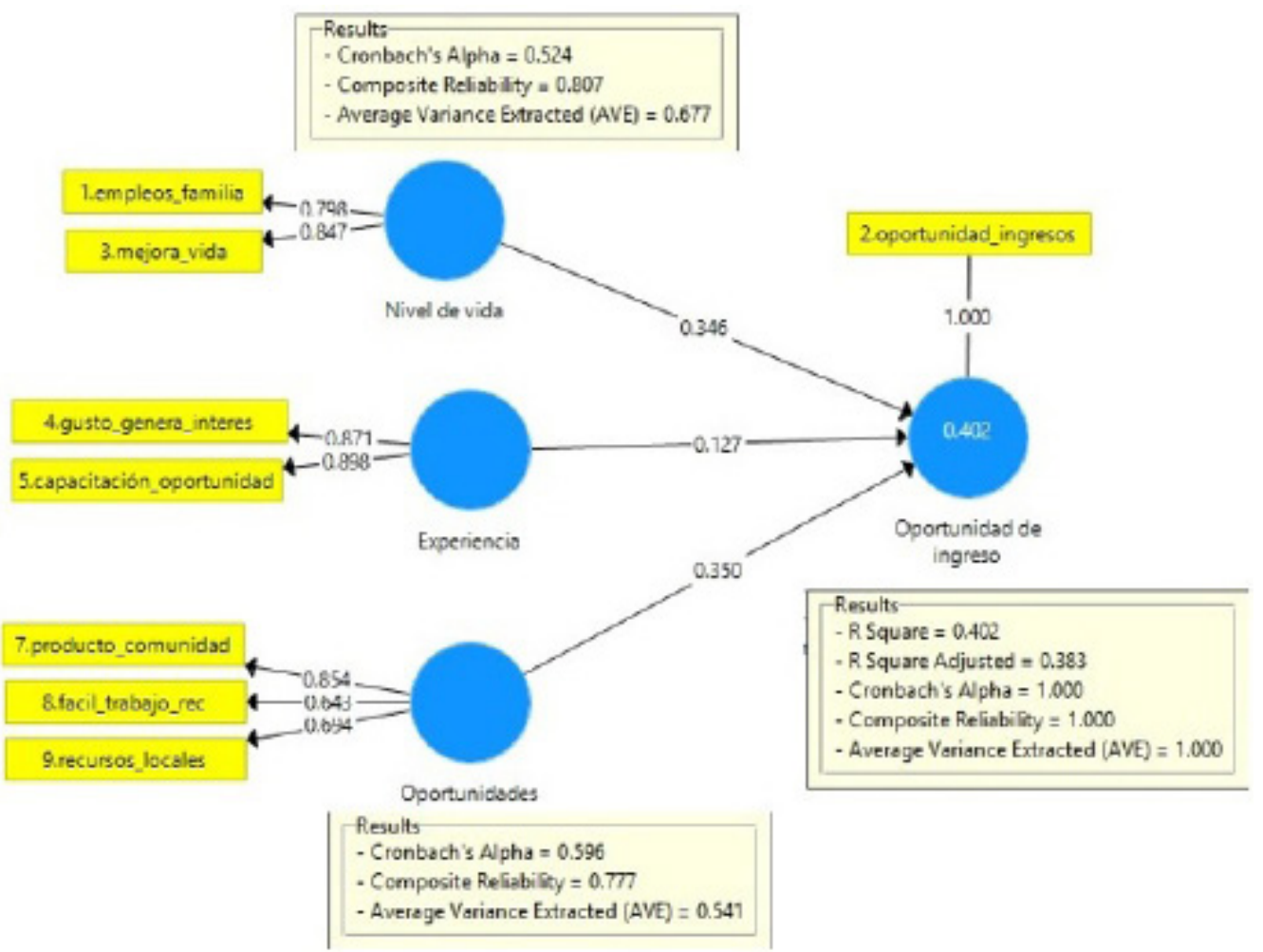

Imagen 1. Modelo diseñado con los estadísticos principales. Fuente Software SmartPLS

5. Validez discriminante (Cada constructo debe ser significativamente diferente del resto de los constructos con los que no se encuentra relacionado según la teoría). Los resultados de la tabla 3 y 4 muestran que cada variable latente es diferente al resto de las demás variables. Esto nos muestra que las variables tienen relación teórica, expresando la correlación entre las variables latentes con sus variables manifiestas. 


\begin{tabular}{|l|c|c|c|c|}
\hline & Experiencia & Nivel de vida & $\begin{array}{c}\text { Oportunidades } \\
\text { de ingreso }\end{array}$ & Oportunidades \\
\hline Experiencia & 0.885 & & & \\
\hline Nivel de vida & 0.516 & 0.823 & & \\
\hline Oportunidades de ingreso & 0.395 & 0.521 & 1.000 & \\
\hline Oportunidades & 0.258 & 0.316 & 0.491 & 0.736 \\
\hline
\end{tabular}

Tabla 3. Criterio Fornell-Larcker con el SmartPLS para la validez discriminante. Elaboración propia.

\begin{tabular}{|l|c|c|c|c|}
\hline & Experiencia & $\begin{array}{c}\text { Nivel de } \\
\text { vida }\end{array}$ & $\begin{array}{c}\text { Oportunidades } \\
\text { de ingreso }\end{array}$ & Oportunidades \\
\hline 1. Empleo familiar & 0.362 & $\mathbf{0 . 7 9 8}$ & $\mathbf{0 . 4 0 1}$ & 0.145 \\
\hline $\begin{array}{l}\text { 2. Oportunidades de in- } \\
\text { gresos }\end{array}$ & 0.395 & $\mathbf{0 . 5 2 1}$ & $\mathbf{1 . 0 0 0}$ & $\mathbf{0 . 4 9 1}$ \\
\hline 3. Mejorar la vida & $\mathbf{0 . 4 8 2}$ & $\mathbf{0 . 8 4 7}$ & $\mathbf{0 . 4 5 5}$ & 0.362 \\
\hline $\begin{array}{l}\text { 4. Gusto por la actividad, } \\
\text { genera interés }\end{array}$ & $\mathbf{0 . 8 7 1}$ & 0.345 & 0.330 & 0.206 \\
\hline $\begin{array}{l}\text { 5. Capacitación da oportuni- } \\
\text { dades }\end{array}$ & $\mathbf{0 . 8 9 8}$ & $\mathbf{0 . 5 5 8}$ & 0.368 & 0.248 \\
\hline $\begin{array}{l}\text { 7. Productos no hay en co- } \\
\text { munidad }\end{array}$ & 0.243 & 0.237 & $\mathbf{0 . 4 6 9}$ & $\mathbf{0 . 8 5 4}$ \\
\hline $\begin{array}{l}\text { 8. Fácil trabajo o pocos re- } \\
\text { cursos }\end{array}$ & -0.011 & 0.145 & 0.198 & $\mathbf{0 . 6 4 3}$ \\
\hline $\begin{array}{l}\text { 9. Aprovechamiento recur- } \\
\text { sos locales }\end{array}$ & 0.249 & 0.297 & 0.342 & $\mathbf{0 . 6 9 4}$ \\
\hline
\end{tabular}

Tabla 3. Criterio Cross loadings con el SmartPLS para la validez discriminante. Elaboración propia.

Resumen de resultados

La investigación arroja un modelo aplicable a las mujeres emprendedoras rurales que tiene el interés de iniciar una empresa, para lo cual tengan aprobado un apoyo de gobierno para el financiamiento del proyecto productivo. Para eso se estudiaron las variables que motivan el emprender un negocio por parte de estas personas.

Los resultados de la investigación incluyen el análisis estadístico de los resultados del cuestionario, así como la comprobación de la hipótesis planteada sobre la que se establece el modelo de implementación para impulsar las motivaciones de emprendedurismo por la mujer rural.

Hipótesis comprobada: Buscar nuevas oportunidades de ingresos por parte de las mujeres rurales en Guanajuato, está en función de mejorar su nivel de vida, experiencia y oportunidades latentes.

$$
“ O p I n g=F(M N V+E X+O P)
$$


De acuerdo con la metodología aplicada y el análisis estadístico de ambas herramientas, se puede comprobar la hipótesis en cuanto a las variables que se relacionan con el modelo planteado:

Relación 1. El emprender para generar empleos dignos para la familia, mejorar las condiciones de vida como salud y educación están relacionadas positivamente con Mejorar el nivel de vida (MNV).

Relación 2. El gusto por una actividad de negocio y la capacitación previa en un oficio positivamente están relacionadas con el la Experiencia (EX).

Relación 3. El hecho que no se ofrece un producto o servicio en la comunidad, el negocio es fácil trabajar o de pocos recursos, y aprovechar los recursos locales tienen relación positivamente con las Oportunidades latentes (OP).

\section{Conclusiones}

Con base en el marco teórico sobre las condiciones de la mujer rural para emprender, la parte empírica sobre el emprendedurismo urbano y rural, los elementos conceptuales que se establecen en otras publicaciones, la información arrojada de la aplicación del programa estatal de capacitación mencionado, hemos establecido de forma preliminar los factores que motivan a la mujer rural para emprender un negocio en su comunidad con el fin de tener un mejor sustento económico y mejor calidad de vida para ella y su familia.

Basados en el análisis de los resultados se puede considerar que las variables latentes presentan los motivos en un modelo comprobado, el cual se compone de las relaciones:

El emprender para generar empleos dignos para la familia, mejorar las condiciones de vida como salud y educación están relacionadas positivamente con Mejorar el nivel de vida (MNV). El gusto por una actividad de negocio y la capacitación previa en un oficio positivamente están relacionadas con el la Experiencia (EX). El hecho que no se ofrece un producto o servicio en la comunidad, el negocio es fácil trabajar o de pocos recursos, y aprovechar los recursos locales tienen relación positivamente con las Oportunidades latentes (OP).

De esta manera se comprueba la hipótesis planeada sobre los elementos conceptuales y que nos muestran los elementos motivadores de la mujer rural para emprender.

Las variables latentes que se han construido se fundamentan en la aplicación del método estadístico utilizado para el análisis y comprobación, no dejando a aspectos intuitivos de los programas de gobierno, además que sobre el estado del arte no se encuentra un fundamento de las mujeres rurales en el estado de Guanajuato. Esto representa el primer paso para emprender por parte de las mujeres en las comunidades.

\section{Recomendaciones}

Los resultados ofrecen un modelo robusto con información del estado de Guanajuato, sobre el cual se puedan trazar líneas estratégicas de motivación para la mujer rural que resulten en proyectos productivos más eficientes y faciliten el emprendedurismo.

Sobre el modelo es necesario medir en algunos programas de gobierno y en forma aislada en proyectos que emprendan las mujeres rurales para validarlo y ser una referencia de conocimiento.

Es necesario ampliar la muestra a todos los municipios del estado para hacerconcluyente el modelo presentado, así como aplicar algunos casos de estudio en los proyectos productivos aprobados por el gobierno de Guanajuato.

Como un estudio posterior complementario, se recomienda ampliar el tema de desarrollo económico con empresas nuevas en las comunidades como un factor de desarrollo rural sustentable que impacte territorialmente con programas integrales. 
Fuentes bibliográficas

Arellano Abasolo, Antonio. (2009). Participación de mujeres en proyectos productivos y cambios en las relaciones de género y las identidades masculinas y femeninas, en Tetela de Ocampo, Puebla. Colpos Digital. Tesis de maestría.

Bonomo Odizzio, A. R. (2013). Mujeres emprendedoras uruguayas en el departamento de San José. Consejo Latinoamericano de Escuelas de Administración.

Borjas Villareal, M. (2014). Acompañamiento en los procesos de capacitación y aprendizaje en mujeres rurales. Colpos Digital.

Buendía-Martínez, I. \& Carrasco, I. (2013). Mujer, actividad emprendedora y desarrollo rural en América Latina y el Caribe. Cuadernos de Desarrollo Rural, 10(72) 21-45.

El Financiero (2014). Empresas cierran por falta de rumbo: IMEF; de 400 nuevas, sobreviven $10 \%$. http:// www.elfinanciero.com.mx/economia/cierrede-empresas-por-falta-de-rumbo-imef-de400-nuevas-sobrevive-10. html, mayo, 2014.

El Economista (2015). México, segundo país a nivel mundial en desarrollo de emprendedores.

http://eleconomista. com.mx/industrias/2015/03/26/mexicosegundo-pais-nivel-mundial-desarrolloemprendedores, marzo, 2015.

Gómez Gutiérrez, Elizabeth Lourdes; Armenteros Acosta, María del Carmen; Guerrero Rampos, Liliana; López Chavarría, Sandra (2015). "Relación entre los perfiles de las mujeres emprendedoras y el acceso a los microcréditos: un análisis en micro empresa de Saltillo, Coahuila," Revista Internacional Administración \& Finanzas, Vol. 8, No. 2, 2015. Martínez Corona, Guadalupe B.; Hartog, Guitte; Parra Inzunza, Filemón; Pedraza López, Josefina. (2009). El programa de la mujer en el sector agrario en tres grupos de mujeres indígenas de Ayotoxco de Guerrero, Puebla. Tesis de maestría. Colegio de Postgraduados, Campus Puebla. Puebla.

Martínez Rivera, Sergio E., Rodríguez Díaz, Luis Fernando. (2013). Emprendedurismo social en México: hacia un modelo de innovación para la inserción social y laboral en el ámbito rural. SEDATU. Estudios Agrarios. May Guillén, A. (2014). Modelo de adición de valor a especies representativas del estado de Tabasco para mujeres rurales y emprendedores. Colpos digital.

Muñoz Aguirre, Christian Dennis (2014). Diagnóstico cualitativo sobre las necesidades de empresarias indígenas y rurales. Inmujeres. ONUDI (2013). Una vía para salir de la pobreza. Organización de las Naciones Unidades para el Desarrollo Industrial.

Pérez Villar, María de los Ángeles (2008). Género, empoderamiento y proyectos productivos: mujeres indígenas chántales de Tabasco. Tesis doctoral Colpos.

Solana-Villanueva, Nélyda, Galmiche-Tejeda, Ángel, \& de los Heros-Rondenil, Martín (2014), https://doi.org/10.29043/liminar.v12i1.328

El Programa de la Mujer Agraria en Tabasco: expectativas y resultados. LiminaR, 12(1) 107124.

Yazmin, T. S. (2010). Necesidades de mujeres jefas de familia. Colpos Digital. 